\title{
Erratum to: Mechanisms for linkage-driven underactuated hand exoskeletons: conceptual design including anatomical and mechanical specifications
}

\author{
Marian Suarez-Escobar ${ }^{1}$ • Juan Andres Gallego-Sanchez ${ }^{1}$. \\ Elizabeth Rendon-Velez ${ }^{1}$ (D)
}

Published online: 27 February 2016

(C) Springer-Verlag France 2016

\section{Erratum to: Int J Interact Des Manuf DOI 10.1007/s12008-015-0297-9}

The authors would like to correct the numbering of the following sections of the text in the publication of the original article:

- The section "Construction of functioning kinematic chain(s)", numbered as Sect. 2.2, should read as a fourth level subtitle. This section is a subsection of Sect. 2.1.3 "The functional requirements" and should read as 2.1.3.1.

- The sections "The structural requirements" and "The design constraints", numbered as Sects. 2.2.1 and 2.2.2, should read as Sects. 2.1.4. and 2.1.5, respectively.

- The sections "Underactuation" and "Link redundancy", numbered as Sects. 2.3 and 2.4, respectively, should read as fourth level subtitles. These sections are subsections of Sect. 2.1.5 "The design constraints" and should read as 2.1.5.1 and 2.1.5.2.

- The sections "Generation of admissible kinematic chains" and "Identification and labeling of compatible kinematic chains", numbered as Sects. 2.5 and 2.6, should read as Sects. 2.2 and 2.3, respectively.

The online version of the original article can be found under doi:10.1007/s12008-015-0297-9.

Elizabeth Rendon-Velez erendonv@eafit.edu.co

Marian Suarez-Escobar msuareze@eafit.edu.co

Juan Andres Gallego-Sanchez jgalleg5@eafit.edu.co

1 Universidad EAFIT, Carrera 49 \# 7 sur-50, 050034 Medellin, Antioquia, Colombia
- The sixth sentence in Sect. 2.2 "Generation of admissible kinematic chains" should read as "Referring to our previously defined structural requirements (Sect. 2.1.4), we are designing finger mechanisms that are planar in nature and have multiple DoFs with only revolute joints". Specifically, note that Sect. 2.2.1 should read as 2.1.4.

- The second sentence in Sect. 2.3 "Identification and labeling of compatible kinematic chains" should read as "This means that, as mentioned in Sect. 2.1.5, to obtain the compatible kinematic chains, the admissible kinematic chains are filtered using the functioning kinematic chain(s), which inherently contains the necessary characteristics to satisfy the design specifications". Specifically, note that Sect. 2.2.2 should read as 2.1.5.

Additionally, the authors would like to correct the spelling of the last name of the second author, which should read as Juan Andres Gallego-Sanchez.

The original article has been updated accordingly. 